\title{
STATIONARY PROBABILITY MEASURES AND TOPOLOGICAL REALIZATIONS
}

\author{
CLINTON T. CONLEY, ALEXANDER S. KECHRIS, \\ AND BENJAMIN D. MILLER
}

\begin{abstract}
We establish the generic inexistence of stationary Borel probability measures for aperiodic Borel actions of countable groups on Polish spaces. Using this, we show that every aperiodic continuous action of a countable group on a compact Polish space has an invariant Borel set on which it has no $\sigma$-compact realization.
\end{abstract}

\section{INTRODUCTION}

It is well known that every standard Borel space is Borel isomorphic to a $\sigma$-compact Polish space (see [Kec95, Theorem 15.6]), and that every Borel action of a countable discrete group on a standard Borel space is Borel isomorphic to a continuous action on a Polish space (see [Kec95, Theorem 13.1]). Here we consider obstacles to simultaneously achieving both results.

In $\S 2$, we give elementary examples of continuous actions of countable groups on Polish spaces which are not Borel isomorphic to continuous actions on $\sigma$-compact Hausdorff spaces. The remainder of the paper is devoted to the implementation of a measure-theoretic strategy for generalizing one of the examples to a much broader context. In $\S 3$, we provide some preliminaries concerning invariant and stationary Borel probability measures. In $\S 4$, we prove our main technical result, a strengthening of Kechris's theorem concerning the generic compressibility of aperiodic countable Borel equivalence relations (see [KM04, Theorem 13.1]). In $\S 5$, we establish the generic inexistence of stationary Borel probability measures. In $\S 6$, we show that every aperiodic continuous action of a countable group on a compact Polish space admits an invariant Borel set on which it is not Borel isomorphic to any continuous action on a $\sigma$-compact Hausdorff space.

2010 Mathematics Subject Classification. Primary 03E15; secondary 28A05.

Key words and phrases. Descriptive set theory, group actions.

The second author was supported in part by NSF Grant DMS-0968710.

The third author was supported in part by SFB Grant 878 . 


\section{EXAMPLES}

Suppose that $X$ is a standard Borel space. We say that a topology $\tau$ on $X$ is compatible with the Borel structure of $X$ if the $\sigma$-algebra generated by $\tau$ is the family of Borel subsets of $X$. Such a $\tau$ is a topological realization of an action $\Gamma \curvearrowright X$ of a topological group if it is Hausdorff and $\Gamma \curvearrowright X$ is $\tau$-continuous.

An equivalence relation is countable if each of its classes is countable. A transversal of an equivalence relation is a set which intersects every equivalence class in exactly one point. We say that a countable Borel equivalence relation on a standard Borel space is smooth if it admits a Borel transversal. While such equivalence relations are typically considered to be simple, even they can be induced by group actions which have no $\sigma$-compact realizations.

Example 1. Consider the action $\mathbb{Z}^{<\mathbb{N}} \curvearrowright \mathbb{Z}^{<\mathbb{N}} \times \mathbb{N}^{\mathbb{N}}$ given by $\gamma \cdot(x, y)=$ $\left(x^{\prime}, y\right)$, where $x^{\prime}(n)=\chi_{U_{n}}(y) \cdot \gamma(n)+x(n)$ and $\left(U_{n}\right)_{n \in \mathbb{N}}$ enumerates a basis for $\mathbb{N}^{\mathbb{N}}$. If $\tau$ is a topological realization of $\mathbb{Z}^{<\mathbb{N}} \curvearrowright \mathbb{Z}^{<\mathbb{N}} \times \mathbb{N}^{\mathbb{N}}$, $n \in \mathbb{N}$, and $\gamma_{n} \in \mathbb{Z}^{<\mathbb{N}}$ is chosen so that $\operatorname{supp}\left(\gamma_{n}\right)=\{n\}$, then

$$
\operatorname{proj}_{\mathbb{N}^{\mathbb{N}}}^{-1}\left(U_{n}\right)=\left\{(x, y) \in \mathbb{Z}^{<\mathbb{N}} \times \mathbb{N}^{\mathbb{N}} \mid \gamma_{n} \cdot(x, y) \neq(x, y)\right\},
$$

so $\operatorname{proj}_{\mathbb{N}^{\mathbb{N}}}$ is $\tau$-continuous, thus $\tau$ cannot satisfy any property preserved under continuous images beyond those satisfied by the topology of $\mathbb{N}^{\mathbb{N}}$. In particular, it is neither $\sigma$-compact nor connected.

Remark 2. The same idea can be used to obtain actions without $\sigma$ compact representations for direct sums of countably many countable abelian groups, and free products of countably many countable groups.

One would really like a free action without a $\sigma$-compact realization, and this particular example is very far from being free. The next observation shows that in the presence of smoothness, this is impossible.

Proposition 3. Suppose that $\Gamma$ is a countable group, $X$ is a standard Borel space, and $\Gamma \curvearrowright X$ is a Borel action, with only countably many stabilizers, whose orbit equivalence relation is smooth. Then $\Gamma \curvearrowright X$ has a $\sigma$-compact Polish realization.

Proof. Fix a Borel transversal $B \subseteq X$ of $E$. Then there is a partition of $B$ into countably many Borel sets $B_{n} \subseteq B$ such that any two points of $B_{n}$ have the same stabilizer $\Gamma_{n}$. The Borel isomorphism theorem (see [Kec95, Theorem 15.6]) yields compact Polish topologies $\tau_{n}$ on $B_{n}$ compatible with the Borel structure of $B_{n}$. Let $\tau_{n}^{\prime}$ denote the natural extension of $\tau_{n}$ to the $\Gamma$-saturation of $B_{n}$ for which the action is continuous, and note that the topologies $\tau_{n}^{\prime}$ can be amalgamated into a single $\sigma$-compact Polish realization of $\Gamma \curvearrowright X$. 
Remark 4. It follows that if $\Gamma$ is a group with only countably many subgroups, then every Borel action $\Gamma \curvearrowright X$ whose induced orbit equivalence relation is smooth has a $\sigma$-compact Polish realization.

A Borel measure $\mu$ on $X$ is invariant with respect to a Borel automorphism $T: X \rightarrow X$ if $\mu(B)=\mu(T(B))$ for all Borel sets $B \subseteq X$. A classical theorem of Krylov-Bogolyubov asserts the existence of invariant Borel probability measures for all homeomorphisms of compact Hausdorff spaces (see Corollary 9 for a more general result).

Example 5. Define $\mathbb{Z}^{<\mathbb{N}} \curvearrowright \mathbb{Z}^{\mathbb{N}}$ by $\gamma \cdot x(n)=\gamma(n)+x(n)$. Suppose, towards a contradiction, that $\tau$ is a $\sigma$-compact realization of $\mathbb{Z}^{<\mathbb{N}} \curvearrowright \mathbb{Z}^{\mathbb{N}}$. Then there is a $\tau$-compact set $K \subseteq \mathbb{Z}^{\mathbb{N}}$ which is non-meager (with respect to the usual topology on $\mathbb{Z}^{\mathbb{N}}$ ). Fix $n \in \mathbb{N}$ and $s \in \mathbb{Z}^{n}$ such that $K$ is comeager in the clopen set $\mathcal{N}_{s}=\left\{x \in \mathbb{Z}^{\mathbb{N}} \mid s \sqsubseteq x\right\}$. Fix $\gamma \in \mathbb{Z}^{<\mathbb{N}}$ with $\operatorname{supp}(\gamma)=\{n\}$. As $\gamma$ is continuous, it sends meager sets to meager sets, so the $\tau$-compact set $L=\left\{x \in \mathbb{Z}^{\mathbb{N}} \mid \forall k \in \mathbb{Z}(k \gamma) \cdot x \in K\right\}$ is non-empty. Then the function $T: L \rightarrow L$ given by $T(x)=\gamma \cdot x$ is a $\tau$-homeomorphism, so there is a $T$-invariant Borel probability measure $\mu$ on $L$. Setting $L_{i}=\{x \in L \mid x(n)=i\}$, it follows that

$$
\mu(L)=\sum_{i \in \mathbb{Z}} \mu\left(L_{i}\right)=\sum_{i \in \mathbb{Z}} \mu\left(T^{i}\left(L_{0}\right)\right)=\sum_{i \in \mathbb{Z}} \mu\left(L_{0}\right),
$$

thus $\mu(L) \neq 1$, a contradiction.

Remark 4 ensures that every Borel action $\mathbb{Z} \curvearrowright X$ whose orbit equivalence relation is smooth has a $\sigma$-compact Polish realization. Nevertheless, there are also actions of $\mathbb{Z}$ without such realizations.

Example 6. Consider the action $\mathbb{Z} \curvearrowright 2^{\mathbb{N}}$ induced by the odometer $\sigma$, i.e., the isometry of $2^{\mathbb{N}}$ sending $(1)^{n \frown}(0)^{\wedge} x$ to $(0)^{n \frown}(1)^{\frown} x$. It is not difficult to see that the usual product measure on $2^{\mathbb{N}}$ is the unique $\sigma$-invariant Borel probability measure on $2^{\mathbb{N}}$. In particular, it follows that if $C \subseteq 2^{\mathbb{N}}$ is a $\sigma$-invariant comeager $\mu$-null Borel set, then there is no $(\sigma\lceil C)$-invariant Borel probability measure. Suppose, towards a contradiction, that $\tau$ is a $\sigma$-compact realization of $\mathbb{Z} \curvearrowright C$. Then there is a $\tau$-compact set $K \subseteq C$ which is non-meager (with respect to the usual topology on $2^{\mathbb{N}}$ ). As $K$ has the Baire property, there exist $n \in \mathbb{N}$ and $s \in 2^{n}$ with the property that $K$ is comeager in the basic clopen set $\mathcal{N}_{s}=\left\{x \in 2^{\mathbb{N}} \mid s \sqsubseteq x\right\}$. Note that the sets of the form $\sigma^{j}\left(\mathcal{N}_{s}\right)$, for $j<2^{n}$, cover $2^{\mathbb{N}}$. As $\sigma$ is a homeomorphism and therefore sends meager sets to meager sets, the $\tau$-compact set $L=\left\{x \in 2^{\mathbb{N}} \mid \forall i \in \mathbb{Z} \exists j<2^{n} \sigma^{i}(x) \in \sigma^{j}(K)\right\}$ is non-empty. As $\sigma$ is a $\tau$-homeomorphism and $L$ is $\sigma$-invariant, there is a $\sigma$-invariant Borel probability measure on $L$, contradicting our choice of $C$. 
Although there are aperiodic Borel automorphisms with multiple invariant Borel probability measures, [KM04, Theorem 13.1] ensures that one can always find a comeager invariant Borel set on which there are no such measures (see also Corollary 14 of $\S 5$ ). As a consequence, one can generalize the last example to aperiodic homeomorphisms of compact Polish spaces which are topologically minimal, in the sense that all of their orbits are dense. As every homeomorphism of a compact Polish space admits an invariant closed set on which it is topologically minimal, every aperiodic such homeomorphism admits an invariant Borel set on which it does not have a $\sigma$-compact realization.

While this approach generalizes to amenable countable groups, the fact that the natural generalization of the Krylov-Bogolyubov theorem fails beyond amenable groups provides a significant barrier to more general results. In what follows, we obtain a generalization by replacing invariance with stationarity.

\section{Existence OF MEASURES}

Suppose that $X$ is a Hausdorff space. A Borel probability measure $\mu$ on $X$ is regular if for all Borel sets $B \subseteq X$ and all $\epsilon>0$, there is an open set $U \subseteq X$ such that $B \subseteq U$ and $\mu(U \backslash B)<\epsilon$. A Borel probability measure $\mu$ on $X$ is invariant with respect to a Borel action $\Gamma \curvearrowright X$ if it is $\gamma$-invariant for all $\gamma \in \Gamma$.

Proposition 7. Suppose that $\Gamma$ is a countable group, $X$ is a compact Hausdorff space, and $\Gamma \curvearrowright X$ is a continuous action with the property that for every finitely generated group $\Delta \leq \Gamma$, there is a $\Delta$-invariant regular Borel probability measure. Then there is a $\Gamma$-invariant regular Borel probability measure.

Proof. Let $C(X, \mathbb{R})$ be the vector space of continuous functions $f: X \rightarrow$ $\mathbb{R}$, and let $C^{*}(X, \mathbb{R})$ be the vector space of linear functionals $\Lambda: C(X, \mathbb{R}) \rightarrow$ $\mathbb{R}$. The weak ${ }^{*}$-topology on $C^{*}(X, \mathbb{R})$ is the coarsest topology rendering the evaluation functions $\Lambda \mapsto \Lambda(f)$ continuous, for all $f \in C(X, \mathbb{R})$. With this topology, $C^{*}(X, \mathbb{R})$ becomes a locally convex (in fact normed) topological vector space.

Let $P(X)$ denote the family of regular Borel probability measures on $X$. We view $P(X)$ as a subset of $C^{*}(X, \mathbb{R})$ by identifying $\mu \in P(X)$ with the functional $f \mapsto \int f d \mu$. The Riesz representation theorem ensures that $P(X)$ coincides with the convex subset of $C^{*}(X, \mathbb{R})$ comprising all positive linear functionals sending the characteristic function of $X$ to 1. It then follows from the Banach-Alaoglu theorem that $P(X)$ is weak*-compact. 
Fix an increasing sequence of finitely generated groups $\Delta_{n}$ with $\Gamma=$ $\bigcup_{n \in \mathbb{N}} \Delta_{n}$. For each $n \in \mathbb{N}$, let $K_{n}$ denote the non-empty compact set of $\Delta_{n}$-invariant regular Borel probability measures. As $K_{n+1} \subseteq K_{n}$ for all $n \in \mathbb{N}$, it follows that the set $K=\bigcap_{n \in \mathbb{N}} K_{n}$ is non-empty, and every $\mu \in K$ is a $\Gamma$-invariant regular Borel probability measure.

The push-forward of a measure $\mu$ on $X$ via a function $f: X \rightarrow Y$ is the measure $f_{*} \mu$ on $Y$ given by $f_{*} \mu(B)=\mu\left(f^{-1}(B)\right)$. The convolution of a probability measure $\varsigma$ on $\Gamma$ with a Borel probability measure $\mu$ on $X$ is the Borel probability measure $\varsigma * \mu$ on $X$ given by $\varsigma * \mu=\int \gamma_{*} \mu d \varsigma(\gamma)$. We say that $\mu$ is $\varsigma$-stationary if $\mu=\varsigma * \mu$. Note that a measure is $\Gamma$ invariant if and only if it is $\varsigma$-stationary with respect to all probability measures $\varsigma$ on $\Gamma$.

We close this section by recalling both the Markov-Kakutani fixed point theorem and its corollary asserting the existence of stationary measures for continuous actions on compact Hausdorff spaces.

Theorem 8 (Markov-Kakutani). Suppose that $K$ is a compact convex subset of a locally convex topological vector space, and $T: K \rightarrow K$ is an affine continuous function. Then there exists $x \in K$ with $T(x)=x$.

Corollary 9. Suppose that $\Gamma$ is a countable group, $X$ is a compact Hausdorff space, $\Gamma \curvearrowright X$ is continuous, and $\varsigma$ is a probability measure on $\Gamma$. Then there is a regular $\varsigma$-stationary Borel probability measure.

Proof. As $\int f d(\varsigma * \mu)=\sum_{\gamma \in \Gamma} \varsigma(\{\gamma\}) \int f \circ \gamma d \mu$, the map $\mu \mapsto \varsigma * \mu$ is weak*-continuous, so Theorem 8 yields a fixed point $\mu$, and any such $\mu$ is a $\varsigma$-stationary regular Borel probability measure.

\section{Paradoxicality}

We use $\operatorname{Pow}(X)$ to denote the family of all subsets of $X$. We say that a function $\phi: X \rightarrow \operatorname{Pow}(Y)$ is strongly injective if it sends distinct points to disjoint sets.

A graph on $X$ is an irreflexive symmetric set $G \subseteq X \times X$. We say that $G$ is locally finite if for all $x \in X$, there are only finitely many $y \in X$ for which $x G y$. We say that $G$ is aperiodic if all of its connected components are infinite. A $G$-barrier for a set $Y \subseteq X$ is a set $Z \subseteq X \backslash Y$ such that the connected component of each point of $Y$ under $G \uparrow(X \backslash Z)$ is finite. Note that $X \backslash Y$ is a $G$-barrier for $Y$ if and only if every connected component of $G \uparrow Y$ is finite.

When $X$ is a standard Borel space and $B \subseteq X$ is a Borel set, we use $[B]^{<\aleph_{0}}$ to denote the family of all finite subsets of $B$, equipped with the standard Borel structure it inherits from $X$. When $E$ is a Borel 
equivalence relation on $X$, we use $[B]_{E}^{<\aleph_{0}}$ to denote the subspace of $[B]^{<\aleph_{0}}$ consisting of those finite sets $F \subseteq B$ for which $F \times F \subseteq E$.

Suppose that $G \subseteq E$ and $\mathscr{D} \subseteq[X]_{E}^{<\aleph_{0}}$. We say that a function $\phi: \mathscr{D} \rightarrow[X]_{E}^{<\aleph_{0}}$ is a $G$-barrier selector if for all $D \in \mathscr{D}$, the set $\phi(D)$ is a $G$-barrier for $D$ contained in $[D]_{E}$. We say that $E$ is $G$-barrier $\mathbb{N}$ paradoxical on $B$ if there is a strongly injective Borel function $\phi: \mathbb{N} \times$ $[B]_{E}^{<\aleph_{0}} \rightarrow[B]_{E}^{<\aleph_{0}}$ such that for all $n \in \mathbb{N}$, the function $\phi_{n}(D)=\phi(n, D)$ is a $G$-barrier selector.

Theorem 10. Suppose that $X$ is a Polish space, $E$ is a countable Borel equivalence relation on $X$, and $G \subseteq E$ is an aperiodic locally finite Borel graph on $X$. Then there is an E-invariant comeager Borel set $C \subseteq X$ on which $E$ is $G$-barrier $\mathbb{N}$-paradoxical.

Proof. We will recursively find Borel sets $\mathscr{D}_{t} \subseteq[X]_{E}^{<\aleph_{0}}$ and strongly injective Borel $G$-barrier selectors $\phi_{t}: \mathscr{D}_{t} \rightarrow\left[X \backslash X_{\sqsubset t}\right]_{E}^{<\aleph_{0}}$, for $t \in \mathbb{N}<\mathbb{N}$, $\mathscr{R}_{t}=\phi_{t}\left(\mathscr{D}_{t}\right), X_{t}=\bigcup \mathscr{R}_{t}, X_{\sqsubset t}=\bigcup_{s \sqsubset t} X_{s}$, and $X_{\sqsubseteq t}=\bigcup_{s \sqsubseteq t} X_{s}$, while ensuring that $X \backslash X_{\sqsubseteq t}$ is a $G$-barrier for $X_{\sqsubseteq t}$ and $[X]_{E}^{<\aleph_{0}}=\bigcup_{n \in \mathbb{N}} \mathscr{D}_{t^{-}(n)}$. We begin by setting $\mathscr{D}_{\emptyset}=\emptyset$ and $\phi_{\emptyset}=\emptyset$.

Suppose now that $t \in \mathbb{N}<\mathbb{N}$ and we have already found $\mathscr{D}_{t}$ and $\phi_{t}$. Let $\mathscr{X}_{t}$ denote the set of all $(D, R, S) \in[X]_{E}^{<\aleph_{0}} \times\left[X \backslash X_{\sqsubseteq t}\right]_{E}^{<\aleph_{0}} \times\left[X \backslash X_{\sqsubseteq t}\right]_{E}^{<\aleph_{0}}$ with the property that $R$ is a $G$-barrier for $D$ and $S$ is a $G$-barrier for $D \cup R$. Let $\mathscr{G}_{t}$ denote the graph on $\mathscr{X}_{t}$ consisting of all distinct $(D, R, S),\left(D^{\prime}, R^{\prime}, S^{\prime}\right) \in \mathscr{X}_{t}$ for which $(D \cup R \cup S) \cap\left(D^{\prime} \cup R^{\prime} \cup S^{\prime}\right) \neq \emptyset$.

Lemma 11. There is a Borel coloring $c_{t}: \mathscr{X}_{t} \rightarrow \mathbb{N}$ of $\mathscr{G}_{t}$.

Proof. Let $\mathscr{G}$ denote the graph on $[X]_{E}^{<\aleph_{0}}$ consisting of all pairs of distinct finite sets with non-empty intersection. By [Mil08, Proposition 4.1], there is a Borel coloring $c:[X]_{E}^{<\aleph_{0}} \rightarrow \mathbb{N}$ of $\mathscr{G}$. Fix a Borel linear ordering $\leq$ of $[X]^{<\aleph_{0}}$, and let $i(D, R, S), j(D, R, S)$, and $k(D, R, S)$ be the unique natural numbers $i, j$, and $k$ such that $D, R$, and $S$ are the $i^{\text {th }}, j^{\text {th }}$, and $k^{\text {th }}$ smallest subsets of $D \cup R \cup S$ with respect to the ordering $\leq$. Then the function $c_{t}: \mathscr{X}_{t} \rightarrow \mathbb{N} \times \mathbb{N} \times \mathbb{N} \times \mathbb{N}$ given by $c_{t}(D, R, S)=(c(D \cup R \cup S), i(D, R, S), j(D, R, S), k(D, R, S))$ yields the desired Borel coloring of $\mathscr{G}_{t}$.

For each $n \in \mathbb{N}$, let $\mathscr{D}_{t^{-}(n)}$ denote the family of sets $D \in[X]_{E}^{<\aleph_{0}}$ for which there exist sets $R, S \in\left[X \backslash X_{\sqsubseteq t}\right]_{E}^{<\aleph_{0}}$ with the property that $(D, R, S) \in \mathscr{X}_{t}$ and $c_{t}(D, R, S)=n$. Define $\phi_{t^{-}(n)}: \mathscr{D}_{t^{-}(n)} \rightarrow[X]_{E}^{<\aleph_{0}}$ by setting $\phi_{t^{-}(n)}(D)=R$ whenever there is $S \in\left[X \backslash X_{\sqsubseteq t}\right]_{E}^{<\aleph_{0}}$ with $(D, R, S) \in \mathscr{X}_{t}$ and $c_{t}(D, R, S)=n$. The definition of $\mathscr{G}_{t}$ ensures that $\phi_{t^{-}(n)}$ is well-defined and strongly injective, while the definition of $\mathscr{X}_{t}$ ensures that $\phi_{t^{\sim}(n)}$ is a $G$-barrier selector and $\mathscr{R}_{t^{\frown}(n)} \subseteq\left[X \backslash X_{\sqsubseteq t}\right]_{E}^{<\aleph_{0}}$. 
To see that $[X]_{E}^{<\aleph_{0}}=\bigcup_{n \in \mathbb{N}} \mathscr{D}_{t^{\frown}(n)}$, note that if $D \in[X]_{E}^{<\aleph_{0}}$ and $R$ is the set of points outside of $D \cup X_{\sqsubseteq t}$ with $G$-neighbors in the connected component of some point of $D$ with respect to $G \uparrow\left(D \cup X_{\sqsubseteq t}\right)$, then $R$ is a $G$-barrier for $D$. Moreover, the fact that $G$ is locally finite and $X \backslash X_{\sqsubseteq t}$ is a $G$-barrier for $X_{\sqsubseteq t}$ ensures that $R$ is finite. Repeating this argument with $D \cup R$ in place of $D$ then yields a finite $G$-barrier $S$ for $D \cup R$, in which case $(D, R, S) \in \mathscr{X}_{t}$. Setting $n=c_{t}(D, R, S)$, it follows that $D \in \mathscr{D}_{t^{\wedge}(n)}$.

It remains to check that $X \backslash X_{\sqsubseteq t^{\frown}(n)}$ is a $G$-barrier for $X_{\sqsubseteq t^{\curvearrowright}(n)}$. Suppose, towards a contradiction, that $\left(x_{i}\right)_{i \in \mathbb{N}}$ is an injective path through $G \uparrow X_{\sqsubseteq t^{\imath}(n)}$. As $X \backslash X_{\sqsubseteq t}$ is a $G$-barrier for $X_{\sqsubseteq t}$, there exists $i \in \mathbb{N}$ such that $x_{i} \notin X_{\sqsubseteq t}$. Fix $(D, R, S) \in \mathscr{X}_{t}$ such that $c(D, R, S)=n$ and $x_{i} \in R$. Then there exists $j>i$ such that $x_{j} \in S$, which contradicts the fact that $S \cap X_{\sqsubseteq t^{\wedge}(n)}=\emptyset$.

This completes the recursive construction. Define $C \subseteq \mathbb{N}^{\mathbb{N}} \times X$ by

$$
C=\left\{(p, x) \in \mathbb{N}^{\mathbb{N}} \times X \mid \forall D \in[x]_{E}^{<\aleph_{0}} \exists^{\infty} n \in \mathbb{N} D \in \mathscr{D}_{p \nmid n}\right\} .
$$

Lemma 12. The set $C$ has a comeager vertical section.

Proof. We will show that, in fact, there are comeagerly many $p \in \mathbb{N}^{\mathbb{N}}$ for which the vertical section $C_{p}$ is comeager. By the Kuratowski-Ulam theorem (see, for example, [Kec95, Theorem 8.41]), it is enough to show that for all $x \in X$, the horizontal section $C^{x}$ is comeager. For this, it is sufficient to check that for all $D \in[x]_{E}^{<\aleph_{0}}$, there are comeagerly many $p \in \mathbb{N}^{\mathbb{N}}$ for which there are infinitely many $n \in \mathbb{N}$ such that $D \in \mathscr{D}_{p \nmid n}$. And for this, it is sufficient to show that for all $k \in \mathbb{N}$, the open set $U_{k}=\left\{p \in \mathbb{N}^{\mathbb{N}} \mid \exists^{\geq k} n \in \mathbb{N} D \in \mathscr{D}_{p \nmid n}\right\}$ is dense.

We proceed by induction on $k$. The base case $k=0$ is trivial, so suppose that $k \in \mathbb{N}$ and we have already established that $U_{k}$ is dense. Given $r \in \mathbb{N}<\mathbb{N}$, fix $s \in \mathbb{N}<\mathbb{N}$ such that $r \sqsubseteq s$ and $\mathcal{N}_{s} \subseteq U_{k}$. Fix $n \in \mathbb{N}$ such that $D \in \mathscr{D}_{s^{\urcorner}(n)}$, set $t=s^{\urcorner}(n)$, and observe that $\mathcal{N}_{t} \subseteq U_{k+1}$, thus $U_{k+1}$ is dense.

Fix $p \in \mathbb{N}^{\mathbb{N}}$ for which $C_{p}$ is comeager, noting that it is also $E$ invariant by the definition of $C$. Define $\phi: \mathbb{N} \times\left[C_{p}\right]_{E}^{<\aleph_{0}} \rightarrow\left[C_{p}\right]_{E}^{<\aleph_{0}}$ by $\phi(k, D)=\phi_{p\lceil n}(D)$, where $n$ is the $k^{\text {th }}$ natural number with $D \in \mathscr{D}_{p\lceil n}$. Then $\phi$ is strongly injective and each of the functions $\phi_{k}(D)=\phi(k, D)$ is a $G$-barrier selector, thus $E$ is $G$-barrier $\mathbb{N}$-paradoxical on $C_{p}$.

$凶$

We say that $E$ is $\mathbb{N}$-paradoxical on $B$ if there is an injective Borel function $\phi: \mathbb{N} \times B \rightarrow B$ such that $\forall n \in \mathbb{N} \forall x \in B x E \phi(n, x)$. 
Corollary 13 (Kechris). Suppose that $X$ is a Polish space and $E$ is an aperiodic countable Borel equivalence relation on $X$. Then there is an E-invariant comeager Borel set $C \subseteq X$ on which $E$ is $\mathbb{N}$-paradoxical.

Proof. By Theorem 10, it is sufficient to show that there is an aperiodic locally finite Borel graph $G \subseteq E$. By the remark at the end of [JKL02, $\S 3.4]$, there is in fact such a graph which generates $E$.

\section{INEXistenCE OF MEASURES}

We say that a Borel measure $\mu$ on $X$ is E-invariant if for every Borel automorphism $T: X \rightarrow X$ whose graph is contained in $E$, the measure $\mu$ is $T$-invariant.

Corollary 14 (Kechris). Suppose that $X$ is a Polish space and $E$ is an aperiodic countable Borel equivalence relation on $X$. Then there is an E-invariant comeager Borel set $C \subseteq X$ which is null with respect to every E-invariant Borel probability measure.

Proof. By appealing to Corollary 13, we obtain an $E$-invariant comeager Borel set $C \subseteq X$ on which $E$ is $\mathbb{N}$-paradoxical. Let $\phi_{n}$ denote the corresponding Borel injections, and observe that if $\mu$ is an $E$-invariant Borel probability measure, then $\mu(C) \geq \sum_{n \in \mathbb{N}} \mu\left(\phi_{n}(C)\right)=\sum_{n \in \mathbb{N}} \mu(C)$, so $\mu(C)=0$.

Identifying $X$ with $[X]_{E}^{1}$, we use our earlier terminology concerning functions on subsets of $[X]_{E}^{<\aleph_{0}}$ for functions on subsets of $X$. We say that $f: B \rightarrow X$ is a choice function for $\phi: B \rightarrow[X]_{E}^{<\aleph_{0}}$ if $f(x) \in \phi(x)$ for all $x \in B$.

Proposition 15. Suppose that $\Gamma$ is a finitely generated infinite group, $S$ is a finite symmetric set which generates $\Gamma$, $\varsigma$ is a probability measure on $\Gamma$ whose support is $S, X$ is a Polish space, $\Gamma \curvearrowright X$ is an aperiodic Borel action, $E$ is the orbit equivalence relation induced by $\Gamma \curvearrowright X$, $G$ is the graph induced by $S \curvearrowright X, B \subseteq X$ is Borel, $\phi: B \rightarrow[X]_{E}^{<\aleph_{0}}$ is a strongly injective Borel $G$-barrier selector, and $\mu$ is a s-stationary Borel measure. Then there is a Borel choice function $f$ for $\phi$ with the property that $\mu(B) \leq \mu(f(B))$.

Proof. We first isolate the feature of $\varsigma$-stationarity relevant to the desired result.

Lemma 16. Suppose that $B \subseteq X$ is a Borel set. If there exists $\gamma \in S$ for which $\mu(B) \neq \gamma_{*} \mu(B)$, then there exists $\delta \in S$ for which $\mu(B)<$ $\delta_{*} \mu(B)$. 
Proof. As the $\varsigma$-stationarity of $\mu$ ensures that $\mu(B)$ is a weighted average of $\left\{\delta_{*} \mu(B) \mid \delta \in S\right\}$, it follows that if some element of this set is distinct from $\mu(B)$, then some element of this set is strictly greater than $\mu(B)$.

By the uniformization theorem for Borel subsets of the plane with countable vertical sections (see, for example, [Kec95, Theorem 18.10]), it is sufficient to produce the desired function off of a $\mu$-null Borel set. In fact, by a measure exhaustion argument, we need only produce the desired function on a $\mu$-positive Borel subset of $B$. As $G$ is locally finite, we can therefore assume that there is a natural number $n$ such that every injective $G$-path of length $n+1$ originating at a point $x \in B$ intersects $\phi(x)$. Setting $\gamma_{s}=\prod_{i<|s|} s(i)$ for $s \in S^{<\mathbb{N}}$, we can moreover assume that for all $i, j \leq n$ and $s \in S^{n}$, the questions of whether $\gamma_{s \nmid i}^{-1} \cdot x \in \phi(x)$ or $\gamma_{s \nmid i}^{-1} \cdot x=\gamma_{s \backslash j}^{-1} \cdot x$ do not depend upon the choice of $x \in B$.

Fix $x \in B$. Then the aperiodicity of $\Gamma \curvearrowright X$ ensures that there is an injective $G$-path $\left(x_{i}\right)_{i \leq n}$ for which $x=x_{0}$. Fix $m \leq n$ with $x_{m} \in \phi(x)$, as well as $s \in S^{m}$ such that $x_{i+1}=s(i)^{-1} \cdot x_{i}$ for all $i<m$, noting that $x_{i}=\gamma_{s\lceil i}^{-1} \cdot x$ for all $i \leq m$.

If $\mu(B)=\left(\gamma_{s}\right)_{*} \mu(B)$, then the function $f(x)=\gamma_{s}^{-1} \cdot x$ is as desired. Otherwise, there is a least natural number $k<m$ for which $\mu(B) \neq$ $\left(\gamma_{s \uparrow(k+1)}\right)_{*} \mu(B)$, in which case our assumption that $S$ is symmetric allows us to recursively appeal to Lemma 16 so as to obtain an extension $t \in S^{n}$ of $s\left\lceil k\right.$ with the property that $\left(\gamma_{t \uparrow i}\right)_{*} \mu(B)<\left(\gamma_{t \uparrow(i+1)}\right)_{*} \mu(B)$ for all $k \leq i<n$. Then the $G$-path $\left(y_{i}\right)_{i \leq n}$ given by $y_{i}=\gamma_{t \uparrow i}^{-1} \cdot x$ is injective, so there exists $\ell \leq n$ for which $y_{\ell} \in \phi(x)$, thus the function $f(x)=\gamma_{t \mid \ell}^{-1} \cdot x$ is as desired.

Remark 17. Although it requires a somewhat more detailed argument, the generalization of Proposition 15 holds in which the symmetry of $S$ is dropped and it generates $\Gamma$ as a semigroup.

Corollary 18. Suppose that $\Gamma$ is a finitely generated infinite group, $X$ is a Polish space, $\Gamma \curvearrowright X$ is an aperiodic Borel action, and $E$ is the orbit equivalence relation induced by $\Gamma \curvearrowright X$. Then there is a comeager E-invariant Borel set $C \subseteq X$ such that for every finite symmetric set $S$ generating $\Gamma$, every probability measure $\varsigma$ on $\Gamma$ whose support is $S$, and every $\varsigma$-stationary Borel probability measure $\mu$, the set $C$ is $\mu$-null.

Proof. Theorem 10 yields a comeager $E$-invariant Borel set $C \subseteq X$ on which $E$ is $G$-barrier $\mathbb{N}$-paradoxical, for all graphs $G$ induced by finite 
symmetric sets $S$ generating $\Gamma$. Given a probability measure $\varsigma$ supported by $S$ and a $\varsigma$-stationary Borel probability measure $\mu$, Proposition 15 yields Borel functions $f_{n}: C \rightarrow C$ with $\mu(C) \geq \sum_{n \in \mathbb{N}} \mu\left(f_{n}(C)\right) \geq$ $\sum_{n \in \mathbb{N}} \mu(C)$, so $\mu(C)=0$.

Remark 19. The same proof actually yields the stronger statement in which $S$ is merely required to be a finite set generating a subgroup $\Delta \leq \Gamma$ (as a semigroup) for which $\Delta \curvearrowright X$ is aperiodic.

\section{TOPOlOGical REALizations}

An action $\Gamma \curvearrowright X$ is topologically minimal if its orbits are dense.

Theorem 20. Suppose that $\Gamma$ is a countable group, $X$ is a compact Polish space, and $\Gamma \curvearrowright X$ is an aperiodic topologically minimal continuous action. Then there is a comeager $\Gamma$-invariant Borel set $C \subseteq X$ such that $\Gamma \curvearrowright C$ has no $\sigma$-compact realization.

Proof. Appeal first to Corollary 14 to obtain a comeager $\Gamma$-invariant Borel set $C \subseteq X$ which is null with respect to every $\Gamma$-invariant Borel probability measure. If there is no finitely generated group $\Delta \leq \Gamma$ for which the union of the finite orbits of $\Delta \curvearrowright X$ is meager, then the set $C$ is already as desired. To see this, suppose, towards a contradiction, that $\tau$ is a $\sigma$-compact realization of $\Gamma \curvearrowright C$.

Lemma 21. Suppose that $K \subseteq C$ is a non-meager $\tau$-compact set. Then there is a comeager $\Gamma$-invariant $\tau$-compact set $L \subseteq \Gamma \cdot K$.

Proof. Fix a non-empty open set $U \subseteq X$ in which $K$ is comeager. Then $U \backslash K$ is meager, so the continuity of $\Gamma \curvearrowright X$ ensures that $\gamma \cdot(U \backslash K)$ is meager for all $\gamma \in \Gamma$, thus so too is the intersection of these sets. It follows that the set $D=C \backslash \bigcup_{\gamma \in \Gamma} \gamma \cdot(U \backslash K)$ is a comeager $\Gamma$-invariant Borel set for which $D \cap U \subseteq D \cap K$. The topological minimality of $\Gamma \curvearrowright X$ yields a finite set $\Lambda \subseteq \Gamma$ with $X=\Lambda \cdot U$, in which case the set $L=\{x \in X \mid \Gamma \cdot x \subseteq \Lambda \cdot K\}$ is $\Gamma$-invariant and $\tau$-compact, and the fact that $D \subseteq L$ ensures that it is comeager.

It follows that there is a comeager $\Gamma$-invariant $\tau$-compact set $K \subseteq C$. Fix an increasing sequence of finitely generated groups $\Delta_{n}$ whose union is $\Gamma$. As $K$ contains a finite orbit of each $\Delta_{n}$, Proposition 7 ensures that there is a $\Gamma$-invariant Borel probability measure supported on $K$, which contradicts our choice of $C$.

It remains to handle the case that there is finitely generated group $\Delta \leq \Gamma$ for which the union of the finite orbits of $\Delta \curvearrowright X$ is meager. Fix a finite symmetric set $S$ generating $\Delta$ and a probability measure $\varsigma$ on $\Gamma$ whose support is $S$. By appealing to Corollary 18, we obtain 
a comeager $\Gamma$-invariant Borel set $D \subseteq C$ which is null with respect to every $\varsigma$-stationary Borel probability measure. Suppose, towards a contradiction, that $\tau$ is a $\sigma$-compact realization of $\Gamma \curvearrowright D$. As Lemma 21 goes through with $D$ in place of $C$, there is a comeager $\Gamma$-invariant $\tau$-compact set $K \subseteq D$. Corollary 9 then ensures that there is a $\varsigma$ stationary Borel probability measure supported on $K$, which contradicts our choice of $C$.

Corollary 22. Suppose that $\Gamma$ is a countable group, $X$ is a compact Polish space, and $\Gamma \curvearrowright X$ is a continuous action whose induced equivalence relation is aperiodic. Then there is a $\Gamma$-invariant Borel set $C \subseteq X$ such that $\Gamma \curvearrowright C$ has no $\sigma$-compact realization.

Proof. As compactness yields a $\Gamma$-invariant closed set $K \subseteq X$ on which $\Gamma \curvearrowright X$ is topologically minimal, Theorem 20 yields the desired result.

Let $\Gamma \curvearrowright 2^{\Gamma}$ denote the shift action given by $\gamma \cdot x(\delta)=x\left(\gamma^{-1} \delta\right)$.

Corollary 23. Suppose that $\Gamma$ is a countably infinite group. Then there is a $\Gamma$-invariant Borel set $B \subseteq 2^{\Gamma}$ with the property that $\Gamma \curvearrowright B$ is free and has no $\sigma$-compact realization.

Proof. By [GJS09, Theorem 1.5], there is a non-empty $\Gamma$-invariant closed set $C \subseteq X$ on which $\Gamma \curvearrowright C$ is free, and an application of Corollary 22 then yields the desired result.

Acknowledgments. We would like to thank Anush Tserunyan for asking the question that motivated this work, Nathaniel Eldredge for useful conversations, and the anonymous referee for several suggestions which helped us improve the clarity of the paper.

Addendum. The idea behind the proof of [KM04, Theorem 13.1] was originally used by Kechris to give a simpler proof of [HK96, Theorem $6.2]$, asserting that every countable Borel equivalence relation is hyperfinite on a comeager Borel set. The latter result was inspired by the earlier [SWW86, Theorem 1.8], a topological special case whose proof is somewhat simpler than even Kechris's later argument. Shortly after submitting this paper, Benjamin Weiss pointed out that there is a simpler proof of a topological special case of Corollary 18 which is sufficient for the proof of the results in $\S 6$.

\section{REFERENCES}

[GJS09] Su Gao, Steve Jackson, and Brandon Seward, A coloring property for countable groups, Math. Proc. Cambridge Philos. Soc. 147 (2009), no. 3, 579-592. MR 2557144 (2010j:03056) 
[HK96] Greg Hjorth and Alexander S. Kechris, Borel equivalence relations and classifications of countable models, Ann. Pure Appl. Logic 82 (1996), no. 3, 221-272. MR 1423420 (99m:03073)

[JKL02] S. Jackson, A. S. Kechris, and A. Louveau, Countable Borel equivalence relations, J. Math. Log. 2 (2002), no. 1, 1-80. MR 1900547 (2003f:03066)

[Kec95] Alexander S. Kechris, Classical descriptive set theory, Graduate Texts in Mathematics, vol. 156, Springer-Verlag, New York, 1995. MR 1321597 (96e:03057)

[KM04] Alexander S. Kechris and Benjamin D. Miller, Topics in orbit equivalence, Lecture Notes in Mathematics, vol. 1852, Springer-Verlag, Berlin, 2004. MR 2095154 (2005f:37010)

[Mil08] Benjamin Miller, The existence of measures of a given cocycle. II. Probability measures, Ergodic Theory Dynam. Systems 28 (2008), no. 5, 16151633. MR 2449547 (2010a:37005)

[SWW86] Dennis Sullivan, B. Weiss, and J. D. Maitland Wright, Generic dynamics and monotone complete $C^{*}$-algebras, Trans. Amer. Math. Soc. 295 (1986), no. 2, 795-809. MR 833710 (87k:46142)

Clinton T. Conley, 584 Malott Hall, Cornell University, Ithaca, NY 14853, USA

E-mail address: clintonc@math.cornell.edu

URL: http://www.math.cornell.edu/People/Faculty/conley.html

Alexander S. Kechris, Mathematics 253-37, Caltech, Pasadena, CA 91125, USA

E-mail address: kechris@caltech.edu

URL: http://www.math.caltech.edu/people/kechris.html

Benjamin D. Miller, Institut für mathematische Logik und Grundlagenforschung, Fachbereich Mathematik und Informatik, Universität Münster, Einsteinstrasse 62, 48149 Münster, Germany

E-mail address: bdm@uni-muenster.de

URL: http://wwwmath.uni-muenster.de/u/ben.miller 